Published by Al-Nahrain College of Medicine P-ISSN 1681-6579

E-ISSN 2224-4719

Email: iraqijms@colmed-alnahrain.edu.iq

http://www.colmed-alnahrain.edu.iq

http://www.iraqijms.net

Iraqi JMS 2018; Vol. 16(1)

\title{
Motor Evoked Potential in Patients with Parkinson's Disease: A Transcranial Magnetic Stimulation Study
}

\author{
Haneen R. Ahmed MSc, Ali F. Al-Hashimi ${ }^{1} P h D$ \\ ${ }^{1}$ Dept. of Physiology and Medical Physics, College of Medicine, Al-Nahrain University, Baghdad, Iraq
}

\begin{abstract}
\end{abstract}
Background

Parkinson's disease (PD) is a neurodegenerative condition of the central nervous system, which is accompanied by the impairment of the cortico-subcortical excitation and inhibition systems. It is characterized by motor and non-motor symptoms, having both hypokinetic and hyperkinetic features.

Objective To investigate the integrity of the central motor pathways by studying the motor evoked potential (MEP) latencies, amplitudes and central conduction time (CMCT) of the median nerve in patients with PD as compared to healthy controls.

Methods Twenty-five patients with documented PD were studied; with a mean age of (63.16 \pm 5.49 years) as compared to 25 age and sex matched apparently healthy controls. All subjects were instructed about the examination and informed consent was provided. Transcranial magnetic stimulation TMS-MEP study of the right median nerve was done. Cortical and cervical latencies and amplitudes of the MEP study were determined. The responses were recorded with both relaxed and slightly contracted target muscle. CMCT calculation was done by subtraction of the latency of peripheral segment of the motor pathway (spinal motor root to muscle) from that of the entire motor pathway (motor cortex to muscle) or by calculation of the CMCT with the F-wave method.

Results The means of the cortical latencies of PD patients during relaxation and facilitation states were lower than controls; and the differences were significant for both ( $\mathrm{P}=0.03$ and 0.02 ; respectively). In both relaxed and facilitation states, the means of CMCT in PD patients were lower than in control and the difference was significant during contraction $(P=0.02)$, and near statistical significance during relaxation $(P=0.08)$. CMCT calculations by the estimation of $F$ wave and distal motor latency (DML) were equivocal between relaxation and facilitation states. Nevertheless, the differences were not statistically significant $(P=0.45 ; P=0.62$; respectively). The means of the MEP amplitude of PD patients were lower than controls $(4.21 \pm 1.94$ versus $4.28 \pm 1.84 \mathrm{mV}$; respectively). Nevertheless, the differences were not significant $(P=0.89)$.

Conclusion Single-pulse TMS is a valuable study to investigate central motor dysfunction in PD. CMCT measurement of the median nerve or any nerve in the upper limb is a potential marker for the evaluation of the severity of PD; especially in the facilitated state.

Keywords Parkinson's disease, TMS, MEP, CL, CMCT

Citation

Ahmed HR, Al-Hashimi AF. Motor evoked potential in patients with Parkinson's Disease: a transcranial magnetic stimulation study. Iraqi JMS. 2018; Vol. 16(1): 14-21. doi: 10.22578/IJMS.16.1.4

List of abbreviations: $A B P=$ Abductor pollicis brevis, $C L C=$ Cortical latency during contracted state, $\mathrm{CLR}=$ cortical latency during relaxed state, CMCT = Central motor conduction time, CMCT-C = Central motor conduction time during contracted states, $\mathrm{CMCT}-\mathrm{F}=$ Central motor conduction time with $\mathrm{F}$ wave, $\mathrm{CMCT}-\mathrm{R}=$ Central motor conduction time during relaxed states, $\mathrm{DML}=$ Distal motor latency, Fmin. = Minimal F-wave latency, MEP = Motor evoked potential, $\mathrm{PD}=$ Parkinson's disease, $\mathrm{PMCT}=$ Peripheral motor conduction time, $\mathrm{SNc}=$ Substantia nigra pars compacta, STN= Subthalamic nucleus, $\mathrm{TMCT}=$ Total motor conduction time, TMS = Transcranial magnetic stimulation

\section{Introduction}

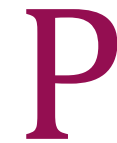
arkinson's

$$
\text { disease }
$$

is

a neurodegenerative condition of the central nervous system (CNS), which is accompanied by the impairment of the corticosubcortical excitation and inhibition systems, hence belonging to the involuntary movement diseases ${ }^{(1)}$. Figures suggest that there are 7-10 million people worldwide who have been diagnosed with PD. Men are 1.5 times more likely than women to develop the disease (2). The core pathology results from the degeneration of dopaminergic neurons in the 
substantia nigra (pars compacta - SNc); where the fibers to the putamen (part of the striatum) are most severely affected. When $80 \%$ of dopamine is being depleted, deficiency in the motor neuron circuitry manifest in the cardinal symptoms of the disease, which include tremor, rigidity, bradykinesia, and postural instability (3). Following degeneration of the SNc dopaminergic neurons projecting to the striatum, several biochemical and electrophysiologic changes occur that cause a characteristic increase in firing rate of the internal globus pallidus (GPi) and the subthalamic nucleus (STN). The consequence of this is inhibition of the thalamo-cortical and brainstem motor systems; reversed by administration of dopaminergic agents ${ }^{(4)}$.

Transcranial magnetic stimulation (TMS) can be regarded as a transcranial electrodeless electric stimulation by electromagnetic induction, because electrical charges flow into an excitable cell membrane, initiating an action potential (5). TMS made it possible for the first time to study the function of the human motor cortex noninvasively and almost painlessly. TMS is now routinely used whenever an objective evaluation of the motor system is required (6); offering a noninvasive and safe approach of stimulating the human motor cortex, and assessing the integrity of the central motor pathways ${ }^{(7)}$.

Of all the different TMS-MEP parameters, the latency of the MEP is generally regarded as the most reliable and useful. If combined with a measure of the peripheral motor conduction time (PMCT), a calculation of the central motor conduction time (CMCT) is possible. In routine clinical practice, this is the most important MEP parameter for evaluation of pyramidal tract function (8). TMS has a series of clinical application in many movement disorders like PD, multiple system atrophy, progressive supranuclear palsy, essential tremor, Huntington's chorea and restless leg syndrome (9). When TMS directed on different levels of the motor system, it will give data about the excitability of the motor cortex, the functional integrity of intracortical neurons, the conduction along corticospinal, corticonuclear, and callosal fibers, as well as the function of nerve roots and peripheral motor path ${ }^{(10)}$.

The aim of the current study was to investigate the integrity of the central motor pathways by studying the MEP of the median nerve and analysis of the cortical latency and CMCT both in relaxed and contracted abductor pollicis brevis (APB) muscle as well as MEP amplitude in patients with PD as compared to healthy controls.

\section{Methods}

Twenty-five patients with documented PD were included in the study; with a mean age of (56.6 \pm 5.6$)$ years as compared to twenty-five age and sex matched apparently healthy controls. All subjects were instructed about the examination and informed consent for participation was provided. The study was approved by the Institute Review Board of the College of Medicine/ Al-Nahrain University.

Each participant will be subjected to TMS-MEP study of the right median nerve, conventional sensory and motor nerve conduction studies to exclude peripheral neuropathy.

The patients with one or more of the following exclusion criteria were eliminated; atypical parkinsonism, secondary parkinsonism, parkinsonism related to other neurodegenerative disease, cerebral and/or medullar pathology, untreated or refractory epilepsy, deep brain stimulation, cardiac pacemaker, prior history of head injury, cranial surgery, stroke, bullet or any implanted electrical biomedical device.

Magnetic stimulation was performed using the (Micromed, 8-channel elecromyograph) EMG /EP machine and Magstim 200 stimulator with the large stimulating coils (type 9784, UK). Subjects were lying down comfortably in a supine position on the couch to guarantee the easy access to the subject's head and they should be seated comfortably to guarantee the easy access to their spine while stimulating them. The subject should be relaxed with eyes 
open. In order to minimize variability in threshold and EP amplitude, it is advised to ask the subject to perform simple mathematical calculations (such as serially adding 7 to 5 or subtracting 9 from 100) (11). MEPs were recorded from the right (APB) muscle by surface electrodes placed on the belly and tendon of the muscle. Stimuli were delivered as single shocks at least 5-10 seconds apart. The electrophysiolgical setting was: high pass filer = $30 \mathrm{~Hz}$, low pass filter $=3000 \mathrm{~Hz}$, time base $=$ $100 \mathrm{~ms}$, and gain $=1 \mathrm{mV} /$ Division $^{(12)}$.

Cortical stimulation of the upper limbs was done by placing the coil tangential to skull, over the vertex in mid-sagittal plane $(C z$, the intersection of the nasion-inion and tragustragus lines) flat on top of the head. The coil edge with maximum magnetic field strength under the middle coil windings thus overlies the motor cortex region for the hand and arm. The direction of the current was clockwise for stimulation of left cortex with the side $A$ of the stimulating coils facing upward. Stimulation was started with about $50 \%$ of maximal stimulator output and then increased until a response with maximum amplitude was registered. To facilitate the response, patients were asked to perform a slight contraction of the target muscle during cortical stimulation. At least three reproducible cortical responses were recorded in order to minimize the variability of the amplitude and latency of the cortical magnetic stimulation ${ }^{(10)}$.

For magnetic stimulation of the cervical root, the center of the coil was placed over $7^{\text {th }}$ cervical spinal process with the subject in the sitting position for the commonly studied hand muscles ${ }^{(13)}$. The circular coil is usually placed in the midline or slightly lateral to this (up to 2 $\mathrm{cm})$ toward the site under investigation. The coil may also be placed lower at the T3 level at $\sim 2 \mathrm{~cm}$ laterally, thus placing the $\mathrm{C} 8 / \mathrm{T} 1$ nerve roots under the upper quadrant of the coil for optimal APB muscle recordings. When using a monophasic stimulator, the current direction is less important for nerve root stimulation compared to cortical stimulation, but a direction of the induced current from medial to lateral has been suggested for both upper and lower extremities, i.e. clockwise orientation of the coil current (looking from behind) for the right side and vice versa for the left (12).

Cortical latency of the TMS-MEP study was determined with activation of target muscle as the shortest interval between time of stimulation and onset of first negative wave of MEP. Recording was both during relaxation and slight muscle contraction ${ }^{(10)}$. The CMCT, which is the latency difference between the MEPs induced by stimulation of the motor cortex and those evoked by spinal (motor root) stimulation. It is calculated by the: Subtraction of the latency of peripheral segment of the motor pathway (spinal motor root to muscle) from that of the entire motor pathway (motor cortex to muscle) so CMCT (ms) = TMCT PMCT; or by calculation of the CMCT with the F-wave method so

CMCT $(\mathrm{ms})=\mathrm{TMCT}-\frac{(\text { Fmin. }+D M L)-1}{2}$

Where

$\mathrm{CMCT}=$ central motor conduction time, $\mathrm{TMCT}=$ total motor conduction time (corticomuscular latency),

Fmin. = minimal F-wave latency,

$\mathrm{DML}=$ distal motor latency,

PMCT = peripheral motor conduction time

(spinal motor root latency) (Wassermann et al. 2008) ${ }^{(10)}$.

Statistical analysis was done using Student's ttest for continuous parameters and Chi-square test for categorical parameters. A p-value $\leq 0.05$ was considered significant (Daniel and Cross, 2013).

Results

Fifty subjects were enrolled in this study; the mean age of those with documented PD $(n=25)$ was (63.16 \pm 5.49 years); comprised (22) males and (3) female as compared to that of 25 healthy controls (65.12 \pm 7.26 years); comprised (23) males and (2) females. There was no significant difference regarding the mean ages and gender between the two studied groups, (Table1). 
Table 1. Demographic characteristics of the studied patients with Parkinson's disease and control groups

\begin{tabular}{cccc}
\hline \multirow{2}{*}{ Parameters } & Patients $(\mathbf{n}=\mathbf{2 5})$ & $\begin{array}{c}\text { Control }(\mathbf{n}=\mathbf{2 5}) \\
\text { mean } \pm \text { SD }\end{array}$ \\
\hline \multirow{2}{*}{ Sex } & Male & 22 & 23 \\
& Female & 3 & 2 \\
\hline \multirow{2}{*}{ Age $(\mathbf{y r})^{*}$} & Mean $\pm S D$ & $63.16 \pm 5.49$ & $65.12 \pm 7.26$ \\
& Range & $47-87$ & $49-76$ \\
\hline
\end{tabular}

* No significant difference between patients and controls using unpaired T-test ( $p$ value $=0.29$ )

Regarding the cortical-latency of the TMS-MEP of the right median nerve was calculated both during relaxed and contracted (facilitated) states. The means of the cortical-latencies of PD patients during relaxation and facilitation states were lower than controls $(22.06 \pm 2.24$ versus $24.12 \pm 4.08$ and $19.28 \pm 2.63$ versus $21.44 \pm 3.72 \mathrm{~ms}$; respectively); and the differences were significant $(\mathrm{P}=0.03$ and 0.02 ; respectively), (Table 2 ).

As in table 2, СMCT was estimated by subtracting the peripheral MCT obtained by cervical spinal roots stimulation from the total MCT obtained by cortical stimulation both during relaxation and facilitation states. In both relaxed and facilitation groups, the means of CMCT in PD patients were lower than in control groups (7.90 \pm 1.66 against $8.69 \pm 1.41$, and $5.28 \pm 1.84$ against $6.58 \pm 1.85 \mathrm{~ms}$; respectively), and the difference was significant during contraction $(P=0.02)$, and near statistical significance during relaxation $(\mathrm{P}=0.08)$, (Table 2).

On the other hand, CMCT calculations by the estimation of $F$ wave and DML were lower in PD patients than their counterparts in the control group for the contracted state; while they were higher during relaxation $(5.34 \pm 2.29$ versus $5.78 \pm 1.71$ and $8.00 \pm 2.1$ versus $7.71 \pm 1.98 \mathrm{~ms}$; respectively). Nevertheless, the differences were not statistically significant ( $P=0.45 ; P=0.62$; respectively), (Table 2 ).

The amplitude of the studied TMS-MEP of the right median nerve was calculated. The means of the MEP amplitude of PD patients were lower than controls $(4.21 \pm 1.94$ versus $4.28 \pm$ $1.84 \mathrm{mV}$; respectively). Nevertheless, the differences were not significant $(P=0.89)$, (Table 2). Figures (1) and (2) shows Right median nerve TMS-MEP parameters both after cortical and peripheral stimulation, recorded from patient with PD and a healthy control; respectively. 
Table 2. Comparison of TMS-MEP study parameters of the right median nerve between patients with Parkinson's disease and control group

\begin{tabular}{|c|c|c|c|}
\hline Parameters & $\begin{array}{c}\text { Patients }(n=25) \\
\text { mean } \pm S D\end{array}$ & $\begin{array}{c}\text { Control }(n=25) \\
\text { mean } \pm S D\end{array}$ & $\begin{array}{c}P \text { value } \\
\text { (unpaired t-test) }\end{array}$ \\
\hline CLR (ms) & $22.06 \pm 2.24$ & $24.12 \pm 4.08$ & 0.03 \\
\hline $\mathrm{CLC}(\mathrm{ms})$ & $19.28 \pm 2.63$ & $21.44 \pm 3.72$ & 0.02 \\
\hline CMCT-R (ms) & $7.90 \pm 1.66$ & $8.69 \pm 1.41$ & 0.08 \\
\hline CMCT-C (ms) & $5.28 \pm 1.84$ & $6.58 \pm 1.85$ & 0.02 \\
\hline CMCT-F-R (ms) & $8.00 \pm 2.10$ & $7.71 \pm 1.98$ & 0.62 \\
\hline CMCT-F-C (ms) & $5.34 \pm 2.29$ & $5.78 \pm 1.71$ & 0.45 \\
\hline MEP Amplitude (mV) & $4.21 \pm 1.94$ & $4.28 \pm 1.84$ & 0.89 \\
\hline
\end{tabular}

$\mathrm{CLR}=$ cortical latency during relaxed state, $\mathrm{CLC}=$ cortical latency during contracted state, $\mathrm{CMCT}-\mathrm{R}=$ central motor conduction time during relaxed states, CMCT-C $=$ central motor conduction time during contracted states, $\mathrm{CMCT}-\mathrm{F}=$ central motor conduction time with $\mathrm{F}$ wave, $\mathrm{MEP}=$ motor evoked potential

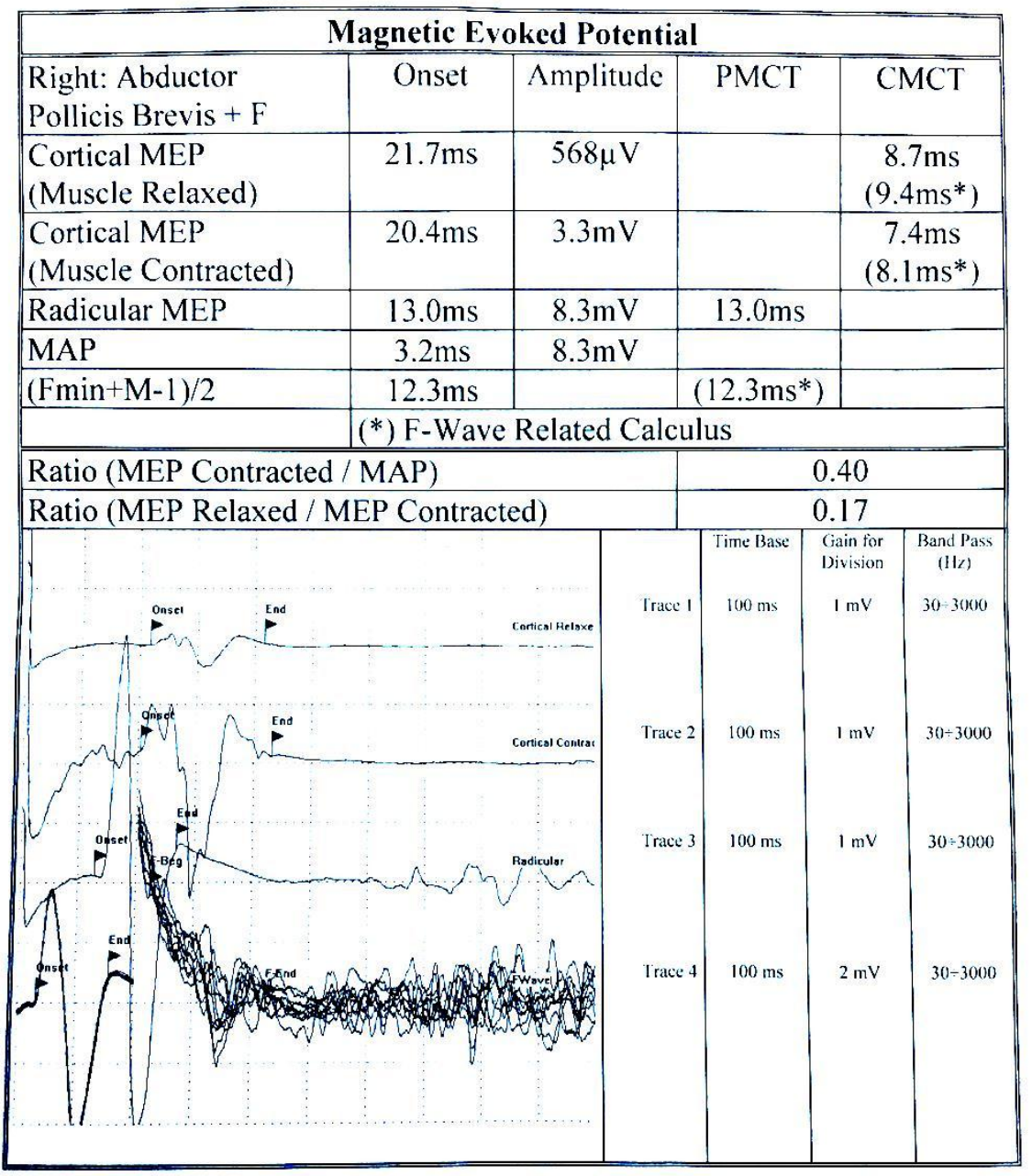

Figure 1. Right median nerve TMS-MEP parameters both after cortical and peripheral stimulation, recorded from patient with Parkinson's disease 


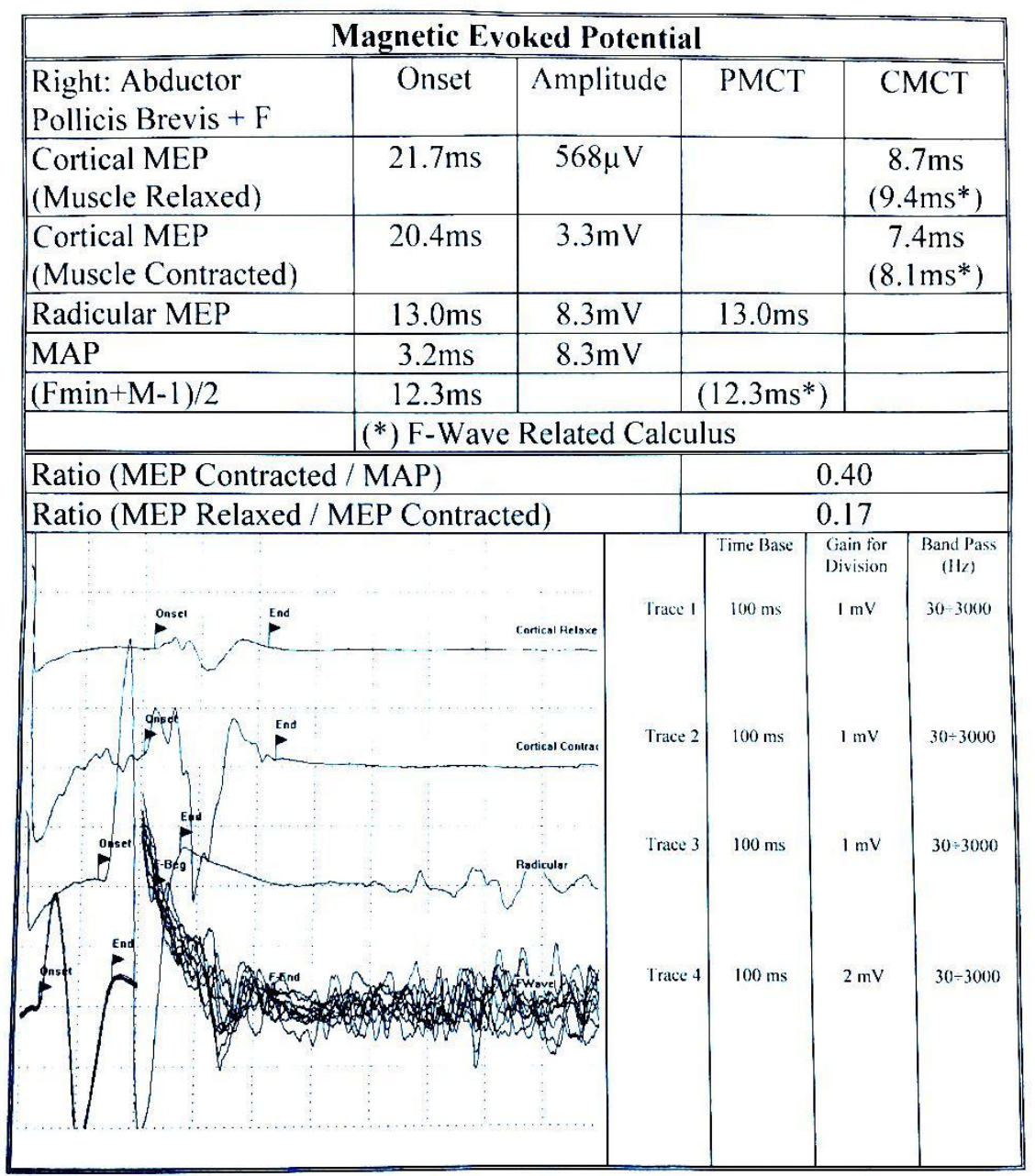

Figure 2. Right median nerve TMS-MEP parameters both after cortical and peripheral stimulation, recorded from a healthy control subject

\section{Discussion}

The TMS is a noninvasive and painless method to stimulate the human brain; it has provided substantial new pathophysiological insights that can be very helpful in providing objective information about the severity of the disease. In the current study, cortical latency of the TMS-MEP study of the right upper limb was calculated both during relaxed and contracted (facilitated) states. Results of the present study showed significantly decreased CLs during both states when compared to controls. This is in agreement with findings of some researchers (14,15); but disagree with others $(16,17)$. Rossini and co-workers in 1999 stated that in patients with PD the cortico-motoneuron conduction was normal ${ }^{(18)}$.
Popa and colleagues stated that their results supported those of previous studies, and that their normal (insignificantly different from controls) values of the MEP latencies and CMCTs in PD patients could be attributed to the fact that the pyramidal tracts are not impaired in pure PD ${ }^{(1)}$.

It is recommended to measure the CMCT while the target muscle contracts at $5 \%$ to $20 \%$ of its maximum strength, because the MEP size saturates for stronger contractions ${ }^{(19)}$. Results of the CMCT calculations in the current study which were recorded both in relaxed and facilitation states were lower than those of the control group and the differences were statistically significant for the contracted state and near statistical significance for the relaxed 
state $(P=0.08)$. This finding is going in accordance with the result of Kandler et al. 1990 and Choi et al. $1999^{(20,21)}$. On the other hand, calculation of CMCT through the estimation of $F$ wave and DML displayed controversial CMCT data; which were lower than their counterparts in the control group for the contracted state; but, higher than the control for the relaxed state. Nevertheless, the differences were not statistically significant.

The reports on TMS have heterogeneous findings. Kandler et al. in 1990 have observed a decrease in CMCT, Choi et al. in $1999(20,21)$ showed that CMCT was shorter in PD patients compared with normal subjects $(P<0.05)$. However, others did not find any difference in CMCT in patients as compared to controls (16). There are only a few studies evaluating the relationship between disease severity, duration of disease and predominant type of PD (akinetic rigid versus tremor dominant) with TMS parameters. Some have reported a decrease in CMCT in patients with predominant rigidity and bradykinesia (20), but others found no difference in any of the TMS parameters, including CMCT in the two broad clinical categories of 'tremor dominant PD' and 'akinetic rigid PD' (16).

MEP amplitude reflects the global excitability of cortical interneurons, corticospinal neurons and spinal motoneurons (22). Results of the present study showed decreased mean amplitude of MEP as compared to healthy controls but with insignificant changes. Several workers have reported increased MEP amplitude at rest in PD patients; which was proposed to be related to an imbalance towards disinhibition in the motor pathway ${ }^{(23)}$. The occurrence of peripheral tremor leads to permanent stimulation of the cerebral cortex. Dopamine secretion diminution is accompanied by intracortical inhibitory mechanism alteration; hence an excessive response to single-pulse stimulation in $\mathrm{PD}$, the consequence of which is the increase of the MEP amplitude (24). However, cortical excitability disturbance is not univocal and unlike healthy subjects, in PD, MEP amplitude increases very little or even decreases further to facilitation (1). Hence, insignificant results of the MEP-amplitude between patients with PD and controls could be most likely expected.

In Conclusion, single-pulse TMS is a valuable study to investigate central motor dysfunction in PD. $C L$ and $C M C T$ measurements of the median nerve or any nerve in the upper limb are potential markers for the evaluation of the severity of PD; especially in the facilitated state. Results of the MEP amplitude are conflicting and require further investigation.

\section{Acknowledgments}

The author would like to thank the members of the Neurophysiology Unit in Al-Imamein AlKadhimein Medical City for their kind support and cooperation; in addition to the members of the Department of Physiology and Medical Physics, College of Medicine, Al-Nahrain University for their encouragement and assistance.

\section{Authors Contribution}

Dr. Ahmed performed the major neurophysiological assessment (TMS-MEP of the Rt. Median N) as well as the conventional sensory and motor nerve conduction study and part of the writing of the manuscript. Dr. AlHashimi participated in the neurophysiological assessment of the patients and controls, analyzing the results and writing the major part of manuscript.

\section{Conflict of interest}

The authors declare no conflict of interest.

Funding

Self-funding.

\section{References}

1. Popa L, Constantinescu A, Popescu CD. Differences of cortical excitability between Parkinson's disease patients and healthy subjects. A comparative TMS study. Romanian J Neurol. 2012; xi(1): 38-43.

2. Barrett $E$, Barman M, Boitano, et al. Ganong's review of medical physiology. 24th ed. New York: McGrawHill Companies; 2012. p. 247. 
3. Carranza M, Snyder MR, Davenport Shaw J, et al. Parkinson's disease - A guide to medical treatment. 1st ed. Torino: SEEd srl; 2013. p. 7.

4. Rubin JE, Mclntyre CC, Turner RS, Wichmann T. Basal ganglia activity patterns in Parkinsonism and computational modeling of their downstream effects. Eur J Neurosci. 2012; 36(2): 2213-28. doi:10.1111/j.1460-9568.2012.08108.x.

5. Andrew BS, Seward RB. The clinical neurophysiology primer. New Jersey: Humana Press Inc. 2007. Chapter 4.

6. Horvath JC, Perez JM, Forrow L, et al. Transcranial magnetic stimulation: A historical evaluation and future prognosis of therapeutically relevant ethical concerns. J Med Ethics. 2011; 37(3): 137-43. doi: 10.1136/jme.2010.039966.

7. Chen R, Cros D, Curra A, et al. The clinical diagnostic utility of transcranial magnetic stimulation- Report of an IFCN committee. Clin Neurophysiol. 2008; 119(3): 504-32. doi: 10.1016/j.clinph.2007.10.014.

8. Kimura J. Electrodiagnosis in disease of nerve and muscle: Principles and practice. 4th ed. USA: Oxford University Press; 2013.

9. Cantello RJ. Applications of transcranial magnetic stimulation in movement disorders. Clin Neurophysiol. 2002; 19(4): 272-293. doi: 10.1097/00004691-200208000-00003.

10. Wassermann EM, Epstein CM, Ziemann U, et al. The motor-evoked potential in health and disease. Oxford handbook of transcranial stimulation. 1st ed. Oxford University Press; 2008. Chapter 19.

11. Rossini PM, Filippi MM, Vernieri F. Neurophysiology of sensorimotor integration in Parkinson's disease. Clin Neurosci. 1998; 5(2): 121-30.

12. Kaddori $H$. Value of transcranial magnetic stimulation and somatosensory evoked potentials versus conventional EMG in the diagnosis of cervical myelopathy. PhD thesis. College of Medicine, AlNahrain University; 2015.

13. Matsumoto $H$, Hanajima R, Terao $Y$, et al. Magneticmotor-root stimulation: Review. Clin Neurophysiol. 2013; 124(6): 1055-67. doi: 10.1016/j.clinph.2012.12.049.

14. Shimamoto $H$, Morimitsu $H$, Sugita $S$, et al. Motor evoked potentials of transcranial magnetic stimulation for Parkinson's disease. No To Shinkei. 1996; Sep; 48(9): 825-9.
15. Bhatia $M$, Johri $S$, Behari $M$. Increased cortical excitability with longer duration of Parkinson's disease as evaluated by transcranial magnetic stimulation. Neurol India. 2003; 51(1): 13-5.

16. Kang JF, Zhang BR, YIN HM, et al. Motor evoked potential in Parkinson's disease by transcranial magnetic stimulation. Chinese J Pathophysiol. 2009; 25(4): 725-8.

17. Livint $L$. Role of functional electrical stimulation and of transcranial magnetic stimulation in improving motor performance in Parkinson's disease. PhD thesis. University of Medicine and Pharmacy of Iași, 2013.

18. Rothwell JC, Hallett M, Berardelli $A$, et al. Magnetic stimulation: motor evoked potentials. The International Federation of Clinical Neurophysiology. Electroencephalogr Clin Neurophysiol Suppl. 1999; 52: 97-103.

19. Ališauskienè $M$, Truffert $A$, Vaičienè $N$, et al. Transcranial magnetic stimulation in clinical practice. Medicina (Kaunas). 2005; 41(10): 813-24.

20. Kandler RH, Jarret JA, Sagar HJ, et al. Abnormalities of central motor conduction in Parkinson's disease. J Neurol Sci. 1990; 100(1-2): 94-7. doi: https://doi.org/10.1016/0022-510X(90)90018-I.

21. Choi J, Park M, Park K, et al. Transcranial magnetic stimulation in Parkinson's disease. J Korean Neurol Assoc. 1999; 17(3): 352-8.

22. Hallett M. Transcranial magnetic stimulation: A primer. Neuron. 2007; 55(2): 187-99. doi: 10.1016/j.neuron.2007.06.026.

23. Ni Z, Chen R. Transcranial magnetic stimulation to understand pathophysiology and as potential treatment for neurodegenerative diseases. Transl Neurodegener. 2015; 4: 22. doi: 10.1186/s40035015-0045-x.

24. Soysal A, Sobe I, Atay $T$, et al. Effect of therapy on motor cortical excitability in Parkinson's disease. Can J Neurol Sci. 2008; 35(2): 166-72.

Correspondence to Dr. Ali F. Al-Hashimi
E-mail: ali_nahrain71@colmed-
alnahrain.edu.iq
Received Apr. 17 2017
Accepted Jul. $12^{\text {th }} 2017$

